\title{
ANALYSIS OF INDUCTIVE FLOW (PRE-FLOW AND POST- FLOW) IN THE VICINITY OF TUBE OPENINGS UNDER MOLECULAR FLOW
}

\author{
MITSURU SESE* \\ Process and Product Development Laboratory, Idemitsu Kosan, Co., Ltd., Sodegaura City, Chiba 299-02 \\ YUJI KAWAMURA \\ Department of Chemical Engineering, Kogakuin University, Tokyo 192
}

Key Words: $\quad$ Rarefied Gas Flow, Molecular Flow, Porpous Media, Flow Properties, Vacuum, Monte Carlo Method

\section{Introduction}

In a previous paper $^{2)}$, the authors analyzed the flow characteristics in a circular tube under free molecular flow and described the so-called "jump phenomena," i.e., the discontinuities in flow properties at both openings of the tube. In that paper, we hypothesized that there exists inductive flow which is responsible for the maintenance of the continuities, but without showing concrete evidence.

In the present paper, we attempt to verify these phenomena by expanding the simulation domain to include the outer space in the vicinity of both tube openings.

\section{Theoretical Considerations}

\subsection{Calculation procedure}

Figure 1 shows the calculation area with an imaginary control surface at the outer space of the tube. To simplify the calculation, symmetric boundaries are selected at the outer space, where the trial molecules reenter from the opposite side during the process in which the molecule collides with the boundary.

The trial molecules are fed at the imaginary inlet plane one after another. We hypothetically set the molecular number density downstream of the imaginary inlet plane to zero.

$$
n=0 \text { at } t=0 \quad x \geq \text { imaginary inlet plane }
$$

The transient variation of the flow properties can be calculated as follows ${ }^{1,2)}$ :

$$
\begin{gathered}
\bar{n}_{i j}{ }^{\tau}=\sum_{\tau=1}^{\tau} f_{i j}{ }^{\tau} /\left(n_{I} V_{i j}\right) \\
\bar{u}_{i j}{ }^{\tau}=\sum_{\tau=1}^{\tau} \sum_{N=1}^{N_{i j}{ }^{\tau}}\left(N f_{i j}{ }^{\tau} u_{N} / \bar{v}\right) / \sum_{N=1}^{N_{i j}{ }^{\tau}} N f_{i j}{ }^{\tau} \\
\bar{F}_{i j}{ }^{\tau}=n_{i j}{ }^{\tau} u_{i j}{ }^{\tau} / F
\end{gathered}
$$

where $\bar{n}_{i j}=n_{i j} / n_{I}, \bar{u}_{i j}=u_{i j} / \bar{v}$, and $F_{i j}=F_{i j} / F . F$ is the * Received February 17, 1993. Correspondence concerning this article should be addressed to $M$. Sese. flux of steady state based on the inlet opening of the tube. The steady-state values of $\bar{n}_{i j}, \bar{u}_{i j}$, and $F_{i j}$ are obtained by ultimate value when $\tau$ is large enough.

\subsection{Calculation conditions}

The conditions for the calculation are as follows: Trial molecules $N=100,000$. Transient time interval $U=$ $10^{-8} \mathrm{sec}$, Radius of tube $R=0.5 \mathrm{~cm}$, and Geometric parameter $L / R=1$.

\section{Results and Discussion}

Figure 2 shows the flow properties, i.e. the molecular number density and superficial velocity, along the flow direction and the jump at the inlet and outlet openings. As can be seen, the cross-sectional averaged values $\left(\bar{n}_{i}, \bar{u}_{i}\right)$ at each location are consistent with each other, even if the calculation domain is changed. Further, although not shown in this paper, the local value at each location $\left(\bar{n}_{i j}, \bar{u}_{i j}\right)$, i.e. the radial distribution, also show the same behavior.

In this method, the calculation proceeds by using molecular velocity from the upper stream (left-hand side) of the two-side Maxwellian. Thus it can be said that the values obtained by this method, are independent of the calculation domain and that the calculated results are appropriate for every calculation. The flow properties continuously vary from the outer space to the tube inlet, and from the tube outlet to the outer space, and show finite values at both openings of the tube, i.e. jump $\Delta$.

Figure 3 and 4 show the radial distribution of local flow properties, including those in the outer space of the tube. The molecular number density continuously decreases from the inlet side $n_{I}$ to the outlet side value $n_{O}$ $=0$. The superficial velocity is accelerated from the inlet side to the outlet side. Figure 5 shows the vector of the molecular number flux (mass velocity) calculated by the product of $n$ and $u$ (Eq. (4)). The vector of the molecular number flux shows inductive flow (pre-flow and postflow) clearly at the outer space in the vicinity of the tube 


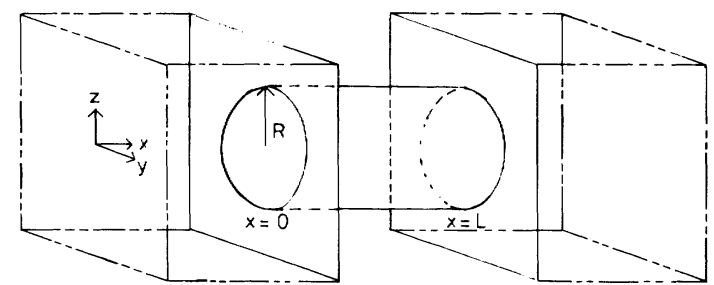

Fig. 1 Calculation area with imaginary control surface

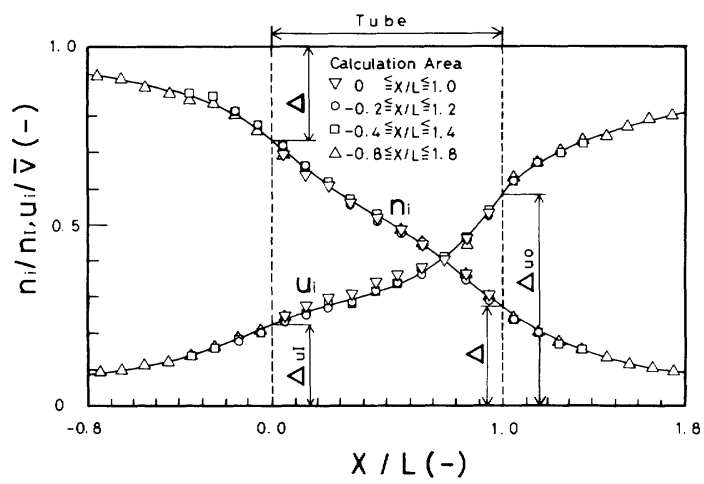

Fig. 2 Molecular number density and superficial average velocity along flow direction

openings respectively.

In summary, it can be concluded that a flow exists that connects the outer space, under equilibrium state with no macroscopic flow, with the tube, under non-equilibrium state with flow. Thus the magnitude of jump $\Delta$ represents the degree of non-equilibrium between the tube under flow and the outer space under equilibrium in the absence of macroscopic flow.

\section{Acknowledgement}

The authors wish to express their sincere appreciation to Prof. Y. Kikuchi, Prof. Y. Nagase and Prof. M. Asaeda, Faculty of Engineering, Hiroshima University, for their invaluable discussions during the early stages of this study.

\section{Nomenclature}

$\begin{array}{ll}F & =\text { molecular number flux } \\ f & =\text { molecular resident probability } \\ L & =\text { length of tube } \\ N & =\text { molecular number } \\ n & =\text { molecular number density } \\ R & =\text { radius of tube } \\ r & =\text { location in radial direction } \\ t & =\text { time } \\ U & =\text { transient time interval } \\ u & =\text { superficial average velocity } \\ V & =\text { volume element } \\ v & =\text { molecular velocity }\end{array}$

[molecules $/ \mathrm{cm}^{2}$ ]<smiles>[AlH2]</smiles>
[molecules $/ \mathrm{cm}^{3}$ ] $[\mathrm{cm}]$ $[\mathrm{cm}]$

$\left[\mathrm{cm}^{3}\right]$

$[\mathrm{cm} / \mathrm{s}]$

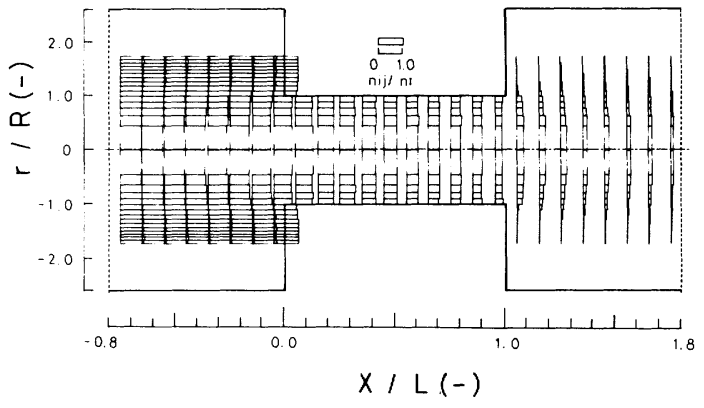

Fig. 3 Radial distribution of molecular number density

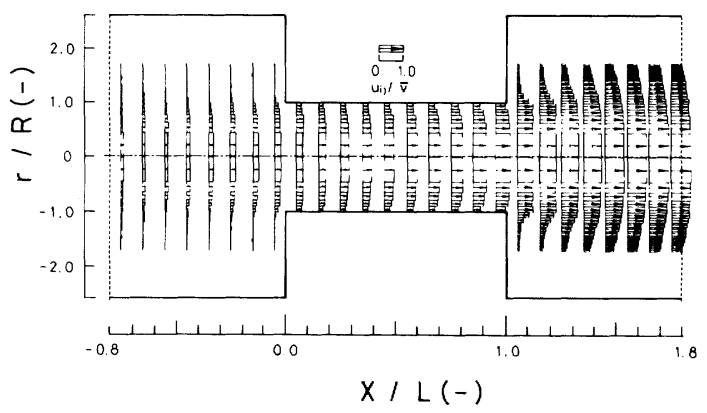

Fig. 4 Radial distribution of superficial average velocity

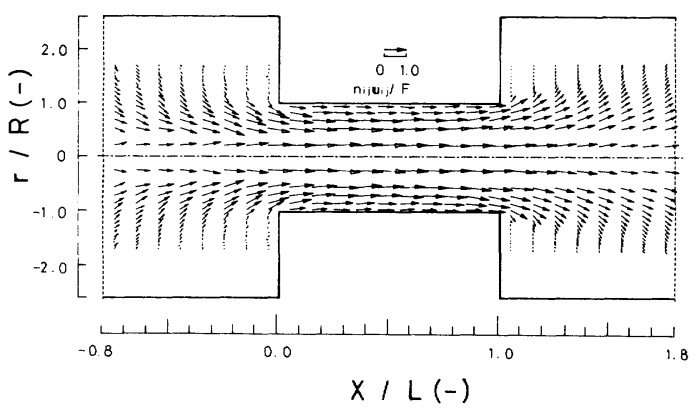

Fig. 5 Vector of molecular number flux (mass velocity)

$x=$ location along flow direction $[\mathrm{cm}]$

$=$ jump

$<$ Subscripts and Superscripts>

$I=$ inlet side of tube

$i=$ increment number along flow direction

$j \quad=\quad$ increment number in radial direction

$O \quad=$ outlet side of tube

$\tau \quad=$ time index

\section{Literature Cited}

1) Kawamura,Y., H.Shinagawa, M. Sese and H. Makihara: J. Chem. Eng. Japan, 10 (2), 104-108 (1977)

2) Sese, M. and Y. Kawamura: J. Chem. Eng. Japan, 23 (2), $137-$ 143 (1990) 